\title{
Electrodeposition Process for the Preparation of Superconducting Thallium Oxide Films
}

\author{
Raghu N. Bhattacharya, Matt Feldmann, David Larbalestier, Richard D. Blaugher
}

\begin{abstract}
A two-layer electrodeposition process was developed to deposit biaxially textured thallium-oxide superconductor films on Ag-coated, single-crystal substrates. Transport measurements for the electrodeposited, thalliumoxide-based superconductor films show values above $10^{6}$ $\mathrm{A} / \mathrm{cm}^{2}$ at $77 \mathrm{~K}$ in zero field. In this paper we are reporting magneto-optical imaging data on electrodeposited 1223 thallium-oxide superconductor films. Magneto-optical imaging provides insight into the nature of current flow in the Tl-oxide superconductor, and thus will help us to improve the critical current density in bulk high-temperature superconductor wire or tape.
\end{abstract}

Index-Electrodeposition, superconductors, magnetooptical image, thallium-oxide.

\section{INTRODUCTION}

$\mathrm{T}$ HE primary technical challenge that must be satisfied to permit high-temperature superconductor (HTS) wire or tape in superconducting magnets or power-related applications is the successful demonstration of a low-cost, high-field, high-current-carrying wire or tape with acceptable mechanical properties. The thallium-based superconducting oxides are excellent candidates because of their high transition temperature reaching to $127 \mathrm{~K}$ and unique features in their growth morphology. Research on the Tl oxides for conductor applications has recently focused on the closely coupled single-layer compounds, such as Tl-1223 phase $\left[(\mathrm{TlPbBi})_{1}(\mathrm{SrBa})_{2} \mathrm{Ca}_{2} \mathrm{Cu}_{3} \mathrm{O}_{\mathbf{x}}\right]$, due to the promise of improved transport behavior in a magnetic field [1-2]. Magnetic field versus temperature irreversibility line for Tl-1223 compares favorably at $77 \mathrm{~K}$ with the $\mathrm{YBaCu}_{3} \mathrm{O}_{\mathrm{x}}(\mathrm{YBCO})$ performance [3]. An additional advantage of $\mathrm{Tl}$-oxide superconductors is short processing time, which can be 10 to 30 minutes compared with many hours to days for other HTS compounds.

In general, the electrodeposition of superconductor tapes for conductor applications has considerable practical

Manuscript received September 16,2000. This work was supported by the U.S. Department of Energy under Contract No. DE-AC36-98G01Q337.

R. N. Bhattacharya is with the National Renewable Energy Laboratory, Golden, CO 80401 USA (telephone: 303-384-6477, e-mail: raghu bhattacharya(a)nrel.gov).

M. Feldmann is with the Applied Superconductivity Center, University of Wisconsin, Madison, WI 53706 USA (telephone: 608-265-5455, e-mail: feldmann(a)cae,wisc.edu)

D. Larbalestier is with the Applied Superconductivity Center, University of Wisconsin, Madison, WI 53706 USA (telephone: 608-263-2194, e-mail: larbales(a)engr.wisc.edu).

R. D. Blaugher is with the National Renewable Energy Laboratory, Golden, CO 80401 USA (telephone: 303-384-6518, e-mail: tichard blaugher@anrel.gov). potential, particularly in the fabrication of large non-planar devices. Electrodeposition is a potentially low-cost nonvacuum process that has the ability to easily deposit superconductor precursor films or tapes at thicknesses up to $15 \mu \mathrm{m}$. Electrodeposited films of all the oxide superconductors have been demonstrated, with the $\mathrm{Tl}$ sytem emerging as most promising [4-6].

In low- $T_{c}$ superconductors [7-8], critical current density $\left(\mathrm{J}_{\mathrm{c}}\right)$ is almost entirely determined by the pinning of vortices at microstructural defects or precipitate in the material (flux pinning). In the HTS, the $J_{c}$ measured by transport over macroscopic lengths is determined not only by intragrain flux pinning at microstructural defects, but also by grain boundary misalignment that influences the connectivity of the superconducting filaments. Some defects in HTS such as cracks and large second-phase particles act as permanent barriers to current flow, while others (e.g., high-angle grain boundaries) exhibit a weak coupling effect that markedly degrades the transport in moderate magnetic fields. This spatially variable electromagnetic coupling produces percolative current flow, meaning that not all of the cross section is useful for carrying transport current. This so-called granular behavior [9] has been visualized by magneto-optical imaging (MOI) in YBCO superconductors. In this paper we are reporting recent studies on the MOI of Tl-oxide superconductors.

\section{EXPERIMENTAL}

The electrodeposited [6] precursor films were obtained by coelectrodeposition of the constituent metals using nitrate salts dissolved in dimethyl sulfoxide (DMSO) solvent. The electrodeposition was performed in a closed-cell configuration at $24^{\circ} \mathrm{C}$ in the presence of dissolved oxygen (oxygen gas was bubbled in the solution during the deposition). A number of electrodeposition runs were performed with different electrolyte compositions, and the precursor films were analyzed by inductively coupled plasma (ICP) spectrometry to establish the stoichiometric ratios of the deposited elements. The cation ratios of the electrodeposition bath were adjusted systematically to obtain (TlBi) $)_{1.1} \mathrm{Sr}_{1.6} \mathrm{Ba}_{0.4} \mathrm{Ca}_{2} \mathrm{Cu}_{4} \quad$ (TBSBCCO) precursor compositions. We increased the $\mathrm{Cu}$ content in the film from 3 to 4, which helped to obtain good-quality films reproducibly. A typical electrolyte-bath composition for the TBSBCCO films consisted of $1 \mathrm{~g} \mathrm{TINO}_{3}, \quad 1 \mathrm{~g}$ $\mathrm{Bi}\left(\mathrm{NO}_{3}\right)_{3} .5 \mathrm{H}_{2} \mathrm{O}, 12.6 \mathrm{~g} \mathrm{Sr}\left(\mathrm{NO}_{3}\right)_{2}, 11.5 \mathrm{~g} \mathrm{Ba}\left(\mathrm{NO}_{3}\right)_{2}, 6.8 \mathrm{~g}$ $\mathrm{Ca}\left(\mathrm{NO}_{3}\right)_{2} .4 \mathrm{H}_{2} \mathrm{O}$, and $2.3 \mathrm{~g} \mathrm{Cu}\left(\mathrm{NO}_{3}\right)_{2} \cdot 6 \mathrm{H}_{2} \mathrm{O}$ dissolved in DMSO solvent. The substrates were single-crystal LAO coated with $300 \AA \mathrm{Ag}$. The films were electroplated by using a constant potential of $-3 \mathrm{~V}$. All samples were electrodeposited in a "vertical cell," in which the electrodes (working, counter, and reference) were suspended vertically 
from the top of the cell. All chemicals were of Analar or Puratronic-grade purity and were used as received. A conventional three-electrode cell was employed, in which the reference electrode was Ag (pseudo-reference) and the counter electrode was a Pt gauze. A Princeton Applied Research potentiostat/galvanostat Model 273A with an IBM PC AT computer interface was used for controlling the pulsedpotential electrolysis and to monitor the current and voltage profiles.

At first, we tried to increase the film thickness by increasing the deposition time. The film thickness did in fact increase with longer deposition time, but the film morphology was poor. A two-layer technique was then tried that used two layers of TBSBCCO films, with an intermediate layer of $\mathrm{Ag}$ to improve the film uniformity. The deposition process is as follows: (a) single-crystal substrates are coated with $300 \AA \mathrm{Ag}$; (b) TBSBCCO films $(0.8$ to $1.3 \mu \mathrm{m})$ are prepared by electrodeposition (ED) on $\mathrm{Ag} / \mathrm{LAO}$; (c) $300 \AA \mathrm{Ag}$ is deposited on ED$\mathrm{TBSBCCO} / \mathrm{Ag} / \mathrm{LAO}$; (d) second layer of TBSBCCO is electrodeposited $(0.8$ to $1.3 \mu \mathrm{m})$ on $\mathrm{Ag} / \mathrm{ED}$ TBSBCCO/Ag/LAO; and the complete two-layer system is reacted.

The samples were characterized by X-ray diffraction including $\theta / 2 \theta$; pole figures; and phi-scans, resistance versus temperature (R-T), four-probe transport, magnetization measurements, scanning electron micrographs (SEM) and by MOI.

\section{RESULTS AND DISCUSSION}

An electrodeposited TBSBCCO precursor film on $300 \AA$ $\mathrm{Ag} / \mathrm{LAO}$, annealed in air at $870^{\circ} \mathrm{C}$ in the presence of a TBSBCCO pellet, shows Tl-1223 phase development [6]. The pole-figure measurement of the (103) HKL peak shows biaxial texture. The omega scan and phi scans indicate the full width at half maximum (FWHM) of only $0.92^{\circ}$ and $0.6^{\circ}$, respectively, which indicate a very high-quality film.

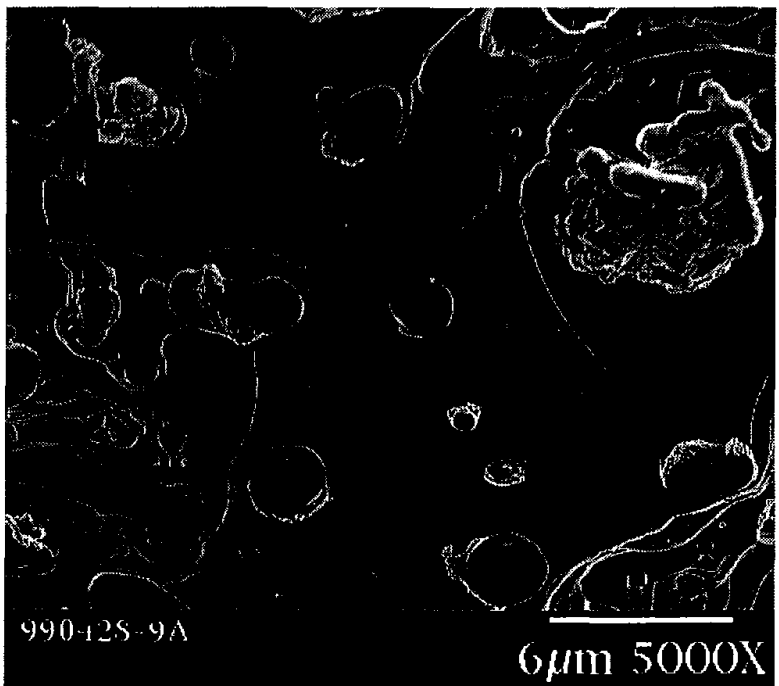

Fig. 1. The SEM of a two-layer annealed ED-TBSBCCO/Ag/EDTBSBCCO/Ag/LAO film.
The SEM analyses (Fig.1) of the presently annealed twolayer film show dense and melted plate-like structure development without any voids. The thickness of the annealed two-layer film varied from 0.8 to $2.6 \mu \mathrm{m}$. The superconductive transition temperature of this film, determined resistively, is about $110 \mathrm{~K}$. Figure 2 shows the critical current density versus magnetic field values at $77 \mathrm{~K}$ of $0.8-\mu \mathrm{m}, 1.6-\mu \mathrm{m}$, and $2.6-\mu \mathrm{m}$ two-layer films. At $77 \mathrm{~K}$ and no magnetic field, the critical current-density value of a two-layer, $0.8-\mu \mathrm{m}$-thick film is $1.1 \times 10^{6} \mathrm{~A} / \mathrm{cm}^{2}$ (Fig.1) using the field criterion of $1 \mu \mathrm{V} / \mathrm{cm}$. The critical current density of the film is calculated using the full cross section of the sample $(3.7 \mathrm{~mm} \times 0.8 \mu \mathrm{m})$. The two-layer, $2.6-\mu \mathrm{m}-$ thick (width $=3.2 \mathrm{~mm}$ ) TBSBCCO film prepared by the electrodeposition process showed critical current $\left(I_{c}\right)$ of 28.24 $A$ at $77 \mathrm{~K}$ (normalized $I_{c}=88.25 \mathrm{~A}$ for $1-\mathrm{cm}$-wide samples). Table 1 summarizes the deposition time, conditions and transport $I_{c}$ of the TBSBCCO films prepared from $1.6 \mu \mathrm{m}$ and $2.6 \mu \mathrm{m}$ single-layer and two-layer electrodeposited precursors. The critical current $\left(I_{\mathfrak{c}}\right)$ of two-layer $E D$ TBSBCCO improved significantly over single-layer TBSBCCO films.

TABLE I

SUMMARY OF DEPOSITED TBSBcco FiLMS AND I

\begin{tabular}{cc}
\hline Deposition time/Conditions & $\begin{array}{c}\text { Normalized } I_{\mathrm{c}} \text { at } 77 \mathrm{~K} \text { (width } \\
\text { normalized to } 1 \mathrm{~cm})\end{array}$ \\
\hline $6 \mathrm{~min} / 1.6 \mu \mathrm{m} /$ Single-layer & $55.70 \mathrm{~A}$ \\
$6 \mathrm{~min} / 1.6 \mu \mathrm{m} /$ Two-layer & $63.10 \mathrm{~A}$ \\
$10 \mathrm{~min} / 2.6 \mu \mathrm{m} /$ Single-layer & $68.80 \mathrm{~A}$ \\
$10 \mathrm{~min} / 2.6 \mu \mathrm{m} /$ Two-layer & $88.25 \mathrm{~A}$ \\
\hline
\end{tabular}

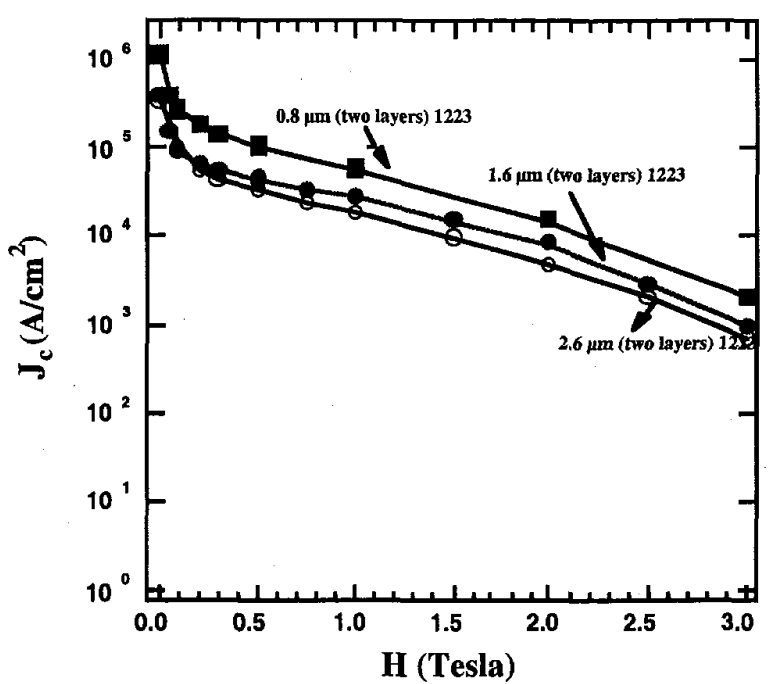

Fig. 2. The magnetic field dependencies of transport $J_{\mathrm{c}}$ at $77 \mathrm{~K}(\mathrm{H} / / \mathrm{c})$ for two-layer 0.8, 1.6, and $2.6 \mu \mathrm{m}$ ED-TBSBCCO/Ag/ED-TBSBCCO/Ag/LAO film.

The MOI data were collected from two-layer $2.6 \mu \mathrm{m}$ EDTBSBCCO films. A representative MOI picture is shown in 
Fig. 3. Even though ED-TBSBCCO films are perfectly biaxially textured films on LAO, MOI data do not reflect the characteristics of the single-crystal LAO substrate. This result is markedly different from the YBCO single-crystal images observed on various substrates such as LAO, YSZ, and STO (Fig, 4). It is argued that the MOI of EDTBSBCCO film is directly related to the unique microstructure that occurs for Tl-oxide films. This microstructure results from the partial melting and recrystallization that occurs during the thallination reaction stage.

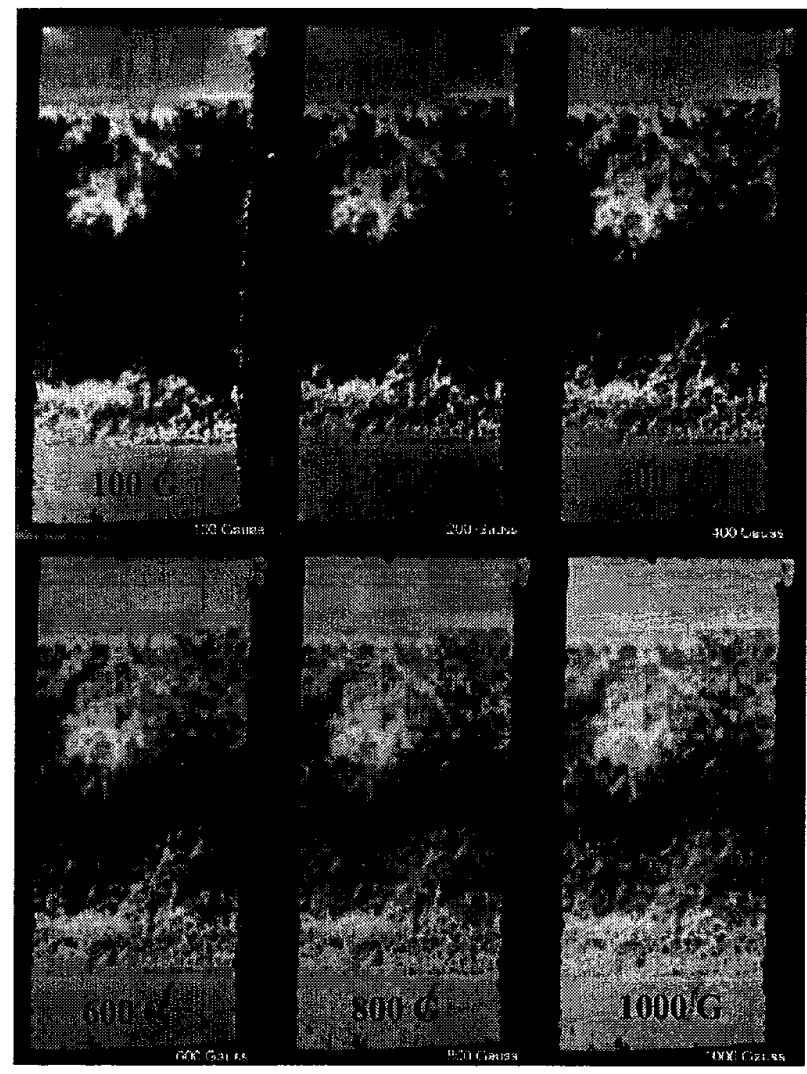

Fig. 3. Magneto-optical imaging of $2.6 \mu \mathrm{m}$ ED-TBSBCCO/Ag/EDTBSBCCO/Ag/LAO film.

The MOI picture of ED-TBSBCCO films is compared in Fig. 5 with PLD YBCO deposited on IBAD YSZ and on rolling assisted biaxially textured (RABiTS) Ni substrates. We cannot make any definitive conclusion from these MOI data but it is clear from Fig. 5 that the observed MOI data for epitaxial Tl-1223 (Fig. 5a) are highly granular and quite similar to the YBCO on IBAD YSZ (Fig. 5b) and RABiTS $\mathrm{Ni}$ (Fig. 5c). These results suggest that the grain-boundary transfer and replication problem seen for YBCO on RABiTS may not be observed for Tl-oxide films processed on similar buffered metallic substrates. Due to the unique "liquidphase" epitaxial processing for the Tl-oxide films, the resulting microstructures for a film on single crystal and RABiTS would be essentially the same. The same basic granular structure would be seen for Tl-oxide superconductors on metallic substrates. Therefore, grain boundary misalignment problems in the substrates may not be replicated for the Tl-oxide superconductors, which may ease the problem for long-length conductor development. Recently, the University of Wisconsin group studied Tloxide superconductor on RABiTS Ni [10]. Their findings suggest that the Ni grain boundaries may in fact be less of a problem for Tl-oxide superconductor than for YBCO. The Tl-oxide superconductor on RABiTS Ni was provided to the Wisconsin group by Judy Wu (University of Kansas). Further studies on Tl-oxide films on RABiTS are needed to fully resolve the advantages of the Tl-oxide superconductor films.

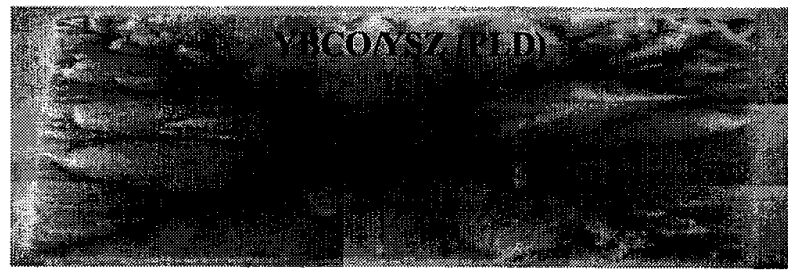

(a)

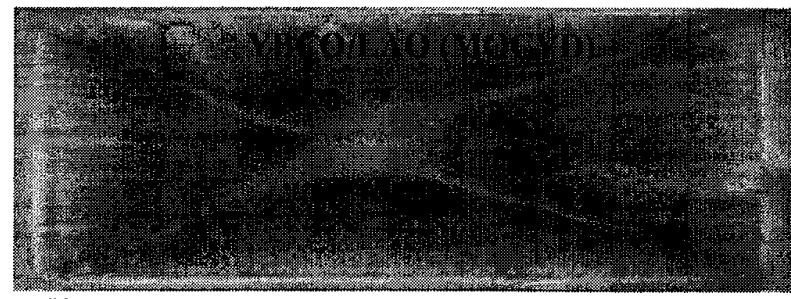

(b)

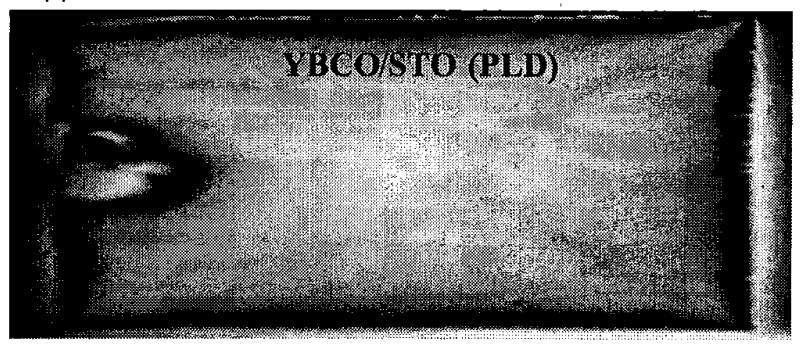

(c)

Fig. 4. Magneto-optical imaging at $8 \mathrm{~K}$ in $800 \mathrm{G}$ field of (a) PLD YBCO on YSZ; (b) MOCVD YBCO on LAO; (c) PLD YBCO on STO.

\section{CONCLUSIONS}

Cathodically electrdeposited Tl-oxide precursor films can be rapidly synthesized with controlled stoichiometry, and upon thallianation, produce high-quality material with promising critical current density. The MOI images on LAO revealed that Tl-oxide superconductor does not exhibit singlecrystal behavior, but shows granular properties similar to YBCO on IBAD YSZ and RABiTS Ni. This leads us to 
believe that grain-boundary misalignment in the substrates may not present any problem for the Tl-oxide superconductor films on metallic substrates, which may ease the problem for very long length conductor development.

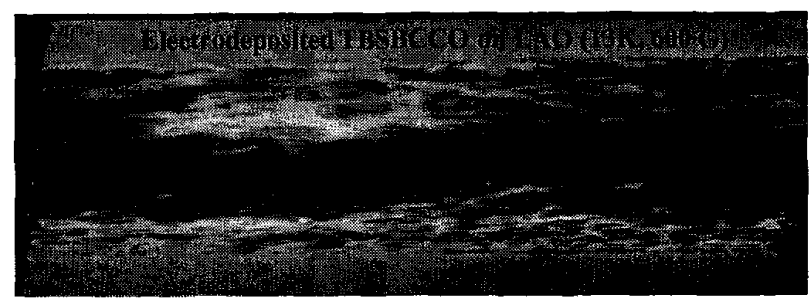

(a)

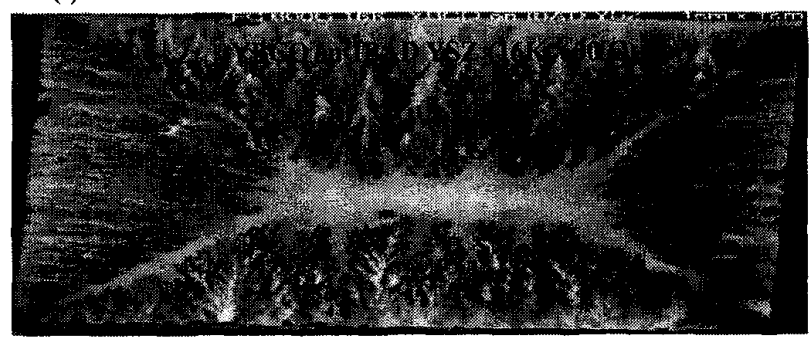

(b)

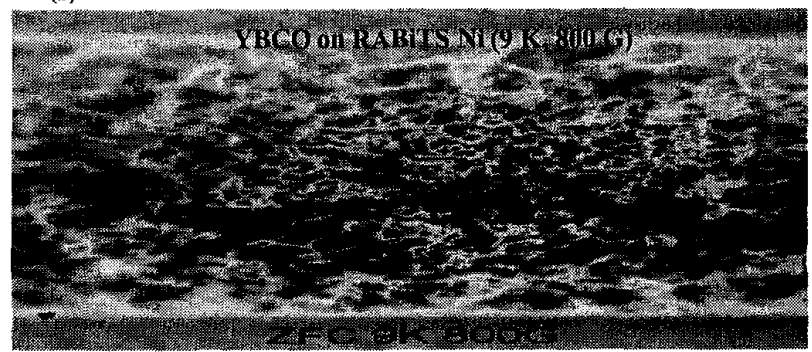

(C)

\section{REFERENCES}

[1] T. J. Doi, T. Yuasa, T. Ozawa, and K. Higashiyama, "Transport critical current densities in uniaxially and biaxially oriented $\mathrm{Tl}_{1}\left(\mathrm{Ba}_{0.8} \mathrm{Sr}_{0.2}\right)_{2} \mathrm{Ca}_{2} \mathrm{Cu}_{3} \mathrm{O}_{9}$ superconductor films on $\mathrm{Ag}$ and $\mathrm{SrTiO}_{3}$ substrates prepared by spray pyrolysis method, "Jpn. J. Appl. Phys., vol. 33 , pp. $5692-5696,1994$.

[2] R. N. Bhattacharya, R. D. Blaugher, A. Natarajan, C. M. Carlson, P. A. Parilla, D. S. Ginley, M. Paranthaman, A. Goyal, and D. M. Kroeger, "Thick-film processing for Tl-oxide wire and tape," Journal of Superconductivity, vol. 11, pp 173-180, 1998.

[3] T. Nabatame, J. Sato, Y. Saito, K. Aihara, T. Kamo, S. Matsuda, "Correlation between irreversibility magnetic fields and the longest $\mathrm{Cu}-\mathrm{O}$ layer spacing in high- $\mathrm{T}_{\mathrm{c}}$ superconductor," Physica $C$, vol. 193, pp. $390-394,1992$.

[4] R. N. Bhattacharya, P. A. Parilla, L. L. Roybal, A. Mason, A. Swartzlander, R. K. Ahrenkiel and R. Noufi, "YbaCuO, PbBiSrCaCu, and $\mathrm{T} I \mathrm{BaCaCuO}$ superconductor thin films via an electrodeposition process," High Temperature Superconducting Compounds III, TMS, pp 169-178, 1991.

[5] R. N. Bhattacharya, R. D. Blaugher, Z. F. Ren, W. Li, J. H. Wang, M. Paranthaman, D. T. Verebelyi, and D. K. Christen, "Superconducting epitaxial (TlBi) ${ }_{0.9} \mathrm{Sr}_{1.6} \mathrm{Ba}_{0.4} \mathrm{Ca}_{2} \mathrm{Cu}_{3} \mathrm{Ag}_{0.2} \mathrm{O}_{\mathrm{x}}$ film from an electrodeposited precursor," Electrochemical and Solid-State Letters, vol. 1, pp 165-167, 1998 .

[6] R. N. Bhattacharya, H. L. Wu, Y. -T. Wang, R. D. Blaugher, S. X. Yang, D. Z. Wang, Z. F. Ren, Y. Tu, D. T. Verebelyi, and D. K. Christen, "Improved electrodeposition process for the preparation of superconducting thallium oxide films," Physica $C$, vol. 333, pp. 59-64, 2000 .

[7] U. Balachandran, A. N. Iyer, P. Haldar, and L. R. Motowado, "Powder-in-tube processing and properties of Bi-2223," JOM., vol. 45, pp 54-58, 1993.

[8] S. X. Dou, and H. K. Liu, "Ag-sheathed $\mathrm{Bi}(\mathrm{Pb}) \mathrm{SrCaCu}$ superconducting tapes," J. Supercond. Sci. Technol., vol. 6, pp $297-$ $314,1993$.

[9] D. Larbalestier, X. Y. Cai, H. Edelman, M. B. Field, Y. Feng, J. Parrell, A. Pashitski, and A. Polyanski, "Visualizing current flow in

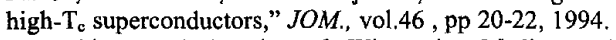

[10] M. Feldmann, University of Wisconsin, Madison, WI, personal communication, 2000.

Fig. 5. Magneto-optical imaging of (a) $2.6 \mu \mathrm{m}$ ED-TBSBCCO/Ag/EDTBSBCCO/Ag/LAO film; (b) PLD YBCO on IBAD YSZ; (c) PLD YBCO on RABiTS Ni. 